\title{
Magnetic resonance imaging of carotid plaques: current status and clinical perspectives
}

\author{
Mohamed Kassem $^{1,2}$, Alexandru Florea ${ }^{2,3}$, Felix M. Mottaghy ${ }^{2,3}$, Robert van Oostenbrugge ${ }^{1,4}$, M. Eline Kooi ${ }^{1,2}$ \\ ${ }^{1}$ CARIM School for Cardiovascular Diseases, Maastricht University, Maastricht, The Netherlands; ${ }^{2}$ Department of Radiology and Nuclear Medicine, \\ Maastricht University Medical Center (MUMC+), Maastricht, The Netherlands; ${ }^{3}$ Department of Nuclear Medicine, University Hospital RWTH \\ Aachen, Aachen, Germany; ${ }^{4}$ Department of Neurology, MUMC+, Maastricht, The Netherlands \\ Contributions: (I) Conception and design: ME Kooi, M Kassem; (II) Administrative support: None; (III) Provision of study materials or patients: \\ None; (IV) Collection and assembly of data: None; (V) Data analysis and interpretation: None; (VI) Manuscript writing: All authors; (VII) Final \\ approval of manuscript: All authors. \\ Correspondence to: Prof. dr. M. Eline Kooi. Department of Radiology and Nuclear Medicine, Maastricht University Medical Centre, P.O. Box 5800, \\ 6202 AZ Maastricht, The Netherlands. Email: eline.kooi@mumc.nl.
}

\begin{abstract}
Rupture of a vulnerable carotid plaque is one of the leading causes of stroke. Carotid magnetic resonance imaging (MRI) is able to visualize all the main hallmarks of plaque vulnerability. Various MRI sequences have been developed in the last two decades to quantify carotid plaque burden and composition. Often, a combination of multiple sequences is used. These MRI techniques have been extensively validated with histological analysis of carotid endarterectomy specimens. High agreement between the MRI and histological measures of plaque burden, intraplaque hemorrhage (IPH), lipid-rich necrotic core (LRNC), fibrous cap (FC) status, inflammation and neovascularization has been demonstrated. Novel MRI sequences allow to generate three-dimensional isotropic images with a large longitudinal coverage. Other new sequences can acquire multiple contrasts using a single sequence leading to a tremendous reduction in scan time. IPH can be easily identified as a hyperintense signal in the bulk of the plaque on strongly $\mathrm{T}_{1}$ weighted images, such as magnetization-prepared rapid acquisition gradient echo images, acquired within a few minutes with a standard neurovascular coil. Carotid MRI can also be used to evaluate treatment effects. Several meta-analyses have demonstrated a strong predictive value of IPH, LRNC, thinning or rupture of the FC for ischemic cerebrovascular events. Recently, in a large meta-analysis based on individual patient data of asymptomatic and symptomatic individuals with carotid artery stenosis, it was shown that IPH on MRI is an independent risk predictor for stroke, stronger than any known clinical risk parameter. Expert recommendations on carotid plaque MRI protocols have recently been described in a white paper. The present review provides an overview of the current status and applications of carotid plaque MR imaging and its future potential in daily clinical practice.
\end{abstract}

Keywords: Atherosclerosis; stroke; carotid artery; magnetic resonance imaging (MRI)

Submitted Feb 21, 2020. Accepted for publication May 10, 2020.

doi: 10.21037/atm-2020-cass-16

View this article at: http://dx.doi.org/10.21037/atm-2020-cass-16

\section{Introduction}

From numerous histopathological studies, it is well known that vulnerable plaque rupture, rather than perfusion defects due to luminal narrowing, is an important cause of stroke (1). Atherosclerosis is a chronic inflammatory disease of the large arteries characterized by the accumulation of lipids in the vessel wall and the formation of fibrous tissue (2). Bifurcations are preferential sites of plaque formation because of the locally reduced wall shear stress (3), which leads to an impaired endothelial function (4). More advanced lesions are characterized by a lipid-rich necrotic core (LRNC), which is separated from 
the lumen by a fibrous cap (FC) (5). Plaques can become increasingly complex, with calcifications, ulcerations, and intraplaque hemorrhage (IPH), thereby increasing the risk to rupture. It is well known that plaque characteristics such as IPH, a large LRNC, and a thin or ruptured fibrous cap (TRFC) are associated with cerebrovascular symptoms. Therefore, diagnostic techniques providing information on the plaque vulnerability have been proposed as more accurate prognostic stratification methods compared to the simple measurement of luminal stenosis. Magnetic resonance imaging (MRI) provides excellent soft tissue contrast, no ionizing radiation, and is not subject to technical challenges, such as shadowing or blooming artefacts caused by calcium deposits. It is also uniquely suited to visualize IPH, which is a strong and independent predictor for stroke (6). MRI is currently recognized as the optimal imaging modality for carotid plaque burden quantification and non-invasive assessment of plaque composition (7). MRI is well validated, highly reproducible and can be used to predict stroke and evaluate treatment effects (8-10). MRI studies also provided more insight in factors that contribute to plaque progression and clinical symptoms, since now, for the first time, the plaque could be followed in time, also before the occurrence of clinical symptoms.

In this review, the ability of MRI to quantify most the important plaque features will be discussed. We will provide an overview of pros and cons of different carotid MRI sequences. The associations of plaque components on MRI with stroke and the predictive value of carotid MRI for stroke will be reviewed. We will summarize the current status of carotid plaque MRI. Moreover, the capability of MRI to measure treatment effects will be elucidated. Additionally, we will discuss novel insights on carotid atherosclerosis that were derived from longitudinal MRI studies. Finally, we will describe new developments and clinical perspectives.

\section{Histopathological evidence on plaque vulnerability}

Numerous histopathological studies have contributed to the concept that rupture of the FC and subsequent thrombosis and embolization is the most important cause of stroke and myocardial infarction $(11,12)$. It is well known that patients with symptomatic carotid artery disease have an enlarged LRNC, and a higher prevalence of a TRFC (13-15). Inflammation, mainly represented by activated macrophages is another hallmark of vulnerable plaques (16-19).
Leaky angiogenic micro-vessels can be a port of entry for inflammatory cells and erythrocytes, leading to further plaque destabilization (20). IPH is also considered as a key factor that is associated with neurological symptoms and is thought to stimulate plaque progression $(3,5,21-25)$. The carotid histological plaque composition in symptomatic patients that underwent CEA is an independent predictor of future cardiovascular events $(26,27)$. Concluding, histopathological studies provided important clues for novel imaging targets, including macrophage-mediated inflammatory changes, neo-angiogenesis, IPH, a large LRNC, and the status of the FC.

\section{MR imaging of carotid plaques}

In the last two decades, MRI was established as the preferred imaging modality to study carotid plaque features (28). High-resolution, multi-contrast carotid MRI can identify and quantify atherosclerotic plaque components (28). The validity of these techniques has been extensively proven using histopathology as a reference standard (Table 1) $(7,8,33)$. Large multicenter MRI studies demonstrated its feasibility (41). Toussaint et al. were the first to show that MRI allows in vivo discrimination of the LRNC, calcifications, and IPH (42). A year later, von Ingersleben et al. confirmed that hemorrhagic regions, calcium, lipid deposits, and fibrous tissue within carotid plaques could be identified using MRI (43). The different plaque components (i.e., LRNC, IPH, calcification, and TRFC) can be distinguished using a combination of various MRI pulse sequences, such as pre- and post-contrast $\mathrm{T}_{1} \mathrm{w}$ turbo-spin echo (44), magnetization-prepared rapid acquisition gradient echo (MPRAGE), and time of flight (TOF) (Figure 1) $(45,46)$. Fat suppression is required to reduce signals from perivascular and subcutaneous adipose tissue. In addition to bright blood MR images to visualize juxtaluminal calcifications, black blood pre-pulses are crucial to optimize contrast between the vessel wall and the lumen. Initially, $\mathrm{T}_{2^{-}}$ weighted MRI $\left(\mathrm{T}_{2} \mathrm{w}\right)$ was used to identify the LRNC (34). Later studies revealed that contrast-enhanced (CE)-MRI enabled improved discrimination of the FC and LRNC compared to conventional $\mathrm{T}_{2} \mathrm{w}$ MRI (47). Ultra-small superparamagnetic iron oxide particles (ferumoxtran-10) can be used to quantify plaque inflammation $(48,49)$. However, this contrast medium is not widely available. Dynamic contrast-enhanced (DCE)-MRI allows to study plaque microvasculature $(50,51)$. 2D black blood sequences are limited by the slice thickness, which hampers 
Table 1 Validation of carotid MRI

\begin{tabular}{|c|c|c|c|}
\hline $\begin{array}{l}\text { Plaque } \\
\text { component }\end{array}$ & MR sequence & $\begin{array}{l}\text { Sensitivity/specificity or correlation with } \\
\text { histology }\end{array}$ & Agreement \\
\hline \multirow[t]{3}{*}{$\mathrm{IPH}$} & MPRAGE & $84 \% / 84 \%(29)$ & \\
\hline & MPRAGE & $93 \% / 96 \%(7)$ & \\
\hline & SNAP versus MPRAGE & & $\kappa=0.82(30)$ \\
\hline LRNC & $\begin{array}{l}T_{2} W \text { (if contrast injection is } \\
\text { contraindicated) }\end{array}$ & $\begin{array}{l}85 \% / 92 \%(34), 90 \% / 84 \%(35), \\
95 \% / 76 \%(36) . \text { Correlation with histology } \\
(r=0.75 ; P<0.001)(36)\end{array}$ & $\begin{array}{l}\text { Inter-reader reproducibility for area } \\
\text { measurements of LRNC (ICC: } 0.92,95 \% \\
\text { CI: } 0.82-0.97)(36)\end{array}$ \\
\hline Calcifications & $\begin{array}{l}\text { Bright blood image and in addition } \\
\text { at least one other weighting }\end{array}$ & With histology $(r=0.74 ; P<0.001)(36)$ & $\begin{array}{l}\text { Inter-observer agreement (ICC: } 0.9 ; 95 \% \\
\mathrm{Cl}: 0.77-0.96) . \text { Agreement with histology } \\
(\kappa=0.75,95 \% \mathrm{Cl}: 0.66-0.84)(36)\end{array}$ \\
\hline \multirow[t]{3}{*}{ Ulceration } & CE-MRA & & $\begin{array}{l}\text { Inter-observer agreement }(\kappa=0.86,95 \% \mathrm{Cl} \text { : } \\
0.77-0.95)(38)\end{array}$ \\
\hline & $\begin{array}{l}\text { TOF (if contrast injection is } \\
\text { contraindicated) }\end{array}$ & TOF: 81\%/90\% (39) & $(\kappa=0.72,95 \% \mathrm{Cl}: 0.58-0.86)(38)$ \\
\hline & $\begin{array}{l}\text { SNAP vs. conventional multi- } \\
\text { contrast }\end{array}$ & & $(\kappa=0.82,95 \% \mathrm{Cl}: 0.65-0.99)(40)$ \\
\hline
\end{tabular}

MRI, magnetic resonance imaging; IPH, intraplaque hemorrhage; LRNC, lipid-rich necrotic core; TRFC, thin or ruptured fibrous cap; MPRAGE, magnetization-prepared rapid acquisition gradient echo; SNAP, simultaneous non-contrast angiography and IPH; TOF, time of flight; CE-MRA, contrast-enhanced MR angiography.

reproducible quantification due to partial volume effects. These challenges were overcome by recent advancements in three-dimensional (3D) sequences, which provide isotropic $3 \mathrm{D}$ images of the entire cervical carotid arteries (52-56) also enabling multi-planar reformatting.

Initially, most studies were performed using 1.5 Tesla MRI. Later, 3.0 Tesla MRI studies were performed to enable improved spatial resolution or better signal-tonoise ratio (SNR) (57-59). Dedicated multi-element carotid radiofrequency MRI coils can be used to acquire images with high SNR and/or high spatial resolution $(60,61)$. This is especially important when visualizing small structures such as the FC. It has been shown that IPH can also be detected using a standard neurovascular coil (45). Recently, novel sequences such as multi-contrast atherosclerosis characterization (MATCH) and simultaneous non-contrast angiography and IPH (SNAP), have been developed that allow the generation of multi-contrast imaging with a single sequence, leading to a tremendous reduction in scan time and resulting in inherent image co-registration $(30,62)$. Recently, expert recommendations on vessel wall MR imaging protocol have been described in white paper (63).

\section{Plaque burden}

Luminal stenosis does not adequately represent plaque burden because of the compensatory enlargement as a response to plaque growth (Glagov remodelling) (64). Therefore, imaging modalities that assess vessel wall dimensions, i.e., plaque burden, provide a more accurate measure of plaque size and severity than current clinically used imaging modalities that only measure carotid artery stenosis. MRI is the most suited imaging technique to quantify plaque burden because its ability to obtain high- 

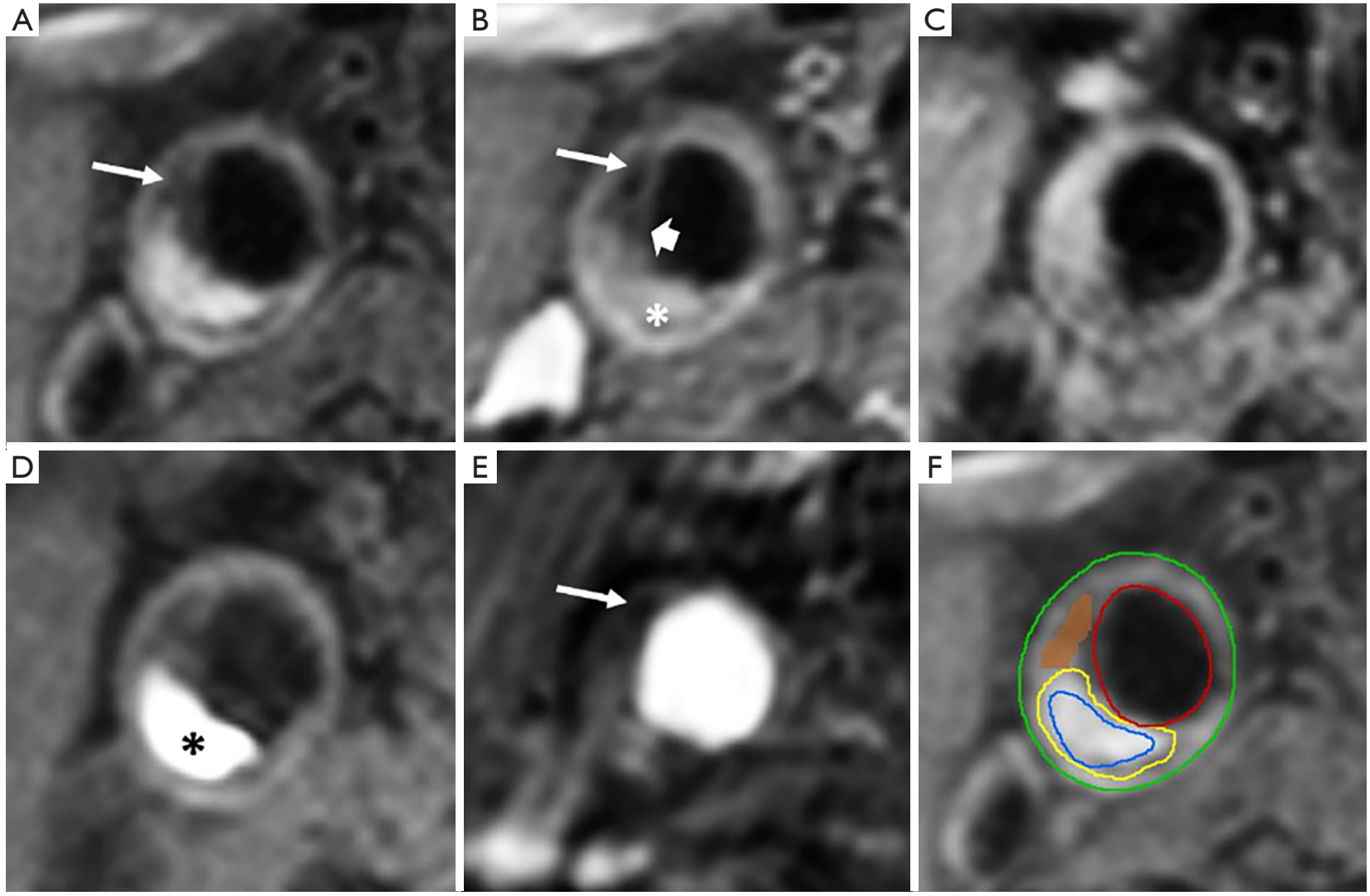

Figure 1 Transversal magnetic resonance (MR) images of a carotid plaque in the right carotid artery with intraplaque hemorrhage (IPH). The following MR sequences were acquired (A) pre-contrast $T_{1} w$-weighted $\left(T_{1} w\right)$ quadruple inversion recovery (QIR) turbo-spin echo (44), (B) post-contrast $\mathrm{T}_{1} \mathrm{w}$ QIR TSE, (C) $\mathrm{T}_{2} \mathrm{w}$ TSE, (D) $\mathrm{T}_{1} \mathrm{w}$ inversion recovery (IR) turbo-field echo (TFE) and (E)time of flight (TOF). A lipid-rich necrotic core LRNC was identified as a region within the bulk of the plaque that does not show contrast enhancement (* on B) with thin and/or ruptured fibrous cap (small arrow on panel B). On the T1 IR-TFE image, a hyper-intense signal in the bulk of the plaque can be clearly observed, indicating the presence of intraplaque hemorrhage IPH within the area of LRNC (* on panel D). Calcification was identified as low signal intensity on TOF and at least two other weightings (long arrow on A, B and E). Panel (F) shows the plaque contours on the pre-contrast $\mathrm{T}_{1} \mathrm{w}$ QIR TSE images (green = outer vessel wall, red = inner vessel wall, yellow = lipid-rich necrotic core, blue = IPH, orange/brown = calcifications).

resolution three-dimensional images with high contrast between the vessel wall, the lumen and the perivascular tissue (Figure 1).

Plaque burden measurements are commonly obtained by subtracting the luminal area from the area that encompasses the outer vessel wall and summing these areas from all MRI slices times the slice thickness, taking into account the slice gap (65). The normalized wall index (NWI), defined as the wall area divided by the total vessel area, was proposed to overcome the different size of carotid arteries in the population (66). It is a very accurate and reproducible measure $(67,68)$.

In the early days, it was shown that slow or turbulent flow can lead to plaque-mimicking artefacts, thereby overestimating the vessel wall (69). Therefore, black blood pre-pulses are required. Early efforts of black blood MRI, using double inversion recovery prepulses in combination with 2D fast-spin echo imaging, revealed that MRI measurements of vessel wall dimensions (wall volume, maximum wall and minimum luminal area) are highly correlated with volumetric measurements of ex vivo CEA specimens (Pearson's $\mathrm{R} \geq 0.90$ ) (70). To obtain black blood images with the double inversion technique, different inversion times are required before and after contrast injection. Therefore, the quadruple inversion recovery technique was developed to acquire black blood MR 
images before and after contrast injection using the same sequence (52). Inversion recovery techniques depend on outflow of blood from the imaging plane in the time period between the inversion pulse(s) and the acquisition of the MRI signal and are therefore problematic in combination with three-dimensional imaging, where thick imaging slabs are excited. Alternative black blood pre-pulses were developed such as multi-slice motion-sensitized drivenequilibrium pre-pulses that do not depend on outflow (69).

Novel 3D black blood sequences such as 3D-MERGE (71) and Delay Alternating with Nutation for Tailored Excitation (DANTE)-prepared 3D MRI (53), offer a high isotropic spatial resolution and the capability to cover the entire cervical carotid arteries. Its short imaging time, ease of use, and ability to accurately assess plaque burden makes these sequences well suited for clinical implementation.

MRI can also be used to quantify the common carotid artery wall dimensions. It was shown that these dimensions correlate well $(\mathrm{r}=0.89, \mathrm{P}<0.001)$ with common carotid intima-media thickness as measured with $\mathrm{B}$-mode ultrasound, however with a much smaller measurement variability for MRI (72). Therefore, when the thickness of the common carotid artery wall is used as a surrogate measure in cardiovascular prevention trials smaller sample sizes and potentially shorter study duration may be possible when using MRI instead of B-mode ultrasound. Recently, it was pointed out by Paraskevas et al., that there is confusion in literature on intima-media thickness (73). Some ultrasound studies have measured the thickness of the far wall of the distal common carotid artery, at a site where there is no plaque, while others included plaque thickness in the measurement of intima-media thickness. The latter method results in an invalid comparison since the vessel wall thickness of patients with and without plaque is a very distinct feature (73).

\section{$I P H$}

To date, IPH is the most widely described predictor of stroke from carotid plaque MRI $(6,46,74)$. The depiction of IPH as a hyper-intense signal compared to surrounding muscle tissue using an MPRAGE sequence (Figure 1) was first presented by Moody et al. (29). Due to relatively short $\mathrm{T}_{1}$ relaxation time of methemoglobin, IPH is hyper-intense on all $\mathrm{T}_{1} \mathrm{w}$ images $(54,75)$. Cappendijk et al. showed a high detection rate $(>80 \%)$ of IPH on MPRAGE images, also known as $\mathrm{T}_{1} \mathrm{w}$ inversion recovery turbo-field echo (IR-TFE) MRI, using histology as a reference standard (76). The IR-
TFE sequence performed superior for the detection of IPH compared to a black blood $\mathrm{T}_{1} \mathrm{w}$ TSE sequence. The interobserver agreement was high for the IR-TFE $(\kappa=0.73)$, while it was low for the T1w TSE sequence $(\kappa=0.35)$. Later, Ota et al. confirmed that MPRAGE has a higher specificity (97\%) and sensitivity ( $80 \%)$ for the detection of IPH compared to fast-spin echo and TOF sequences (77). Semiautomatic quantification of IPH volume on MRI has shown to correlate well with histology $(54,75,78,79)$. IPH can also be identified on contrast-enhanced MR angiography (CE-MRA) mask images (80). Recently, a multi-contrast sequence, 3D-SNAP, was developed to detect lumen stenosis and IPH with a single sequence with inherent image co-registration. Its performance for the identification of IPH was comparable with MPRAGE ( $\kappa=0.82$ ) (30). Alternatively, another multi-contrast sequence, MATCH simultaneously obtains 3 different contrast weightings (hyper- $\mathrm{T}_{1} \mathrm{w}, \mathrm{T}_{1} \mathrm{w}$, and gray blood) in a 5 -minute scan to image IPH, the LRNC and calcifications (62). These novel multi-contrast sequences may represent alternatives for multi-sequence MR imaging. However, larger studies to validate MATCH are required. A meta-analysis on the diagnostic performance of MRI for detecting IPH in the carotid arteries revealed excellent specificity (92\%) and good sensitivity (87\%) (31). Moreover, carotid plaque $\mathrm{T}_{1}$ mapping has been developed to obtain more quantitative, reproducible measurements of IPH $(81,82)$.

\section{LRNC}

The LRNC has a short transverse relaxation time $\left(\mathrm{T}_{2}\right)$ compared to the surrounding fibrous tissue (83). Therefore, the LRNC is detected as hypo-intense on $\mathrm{T}_{2} \mathrm{w}$ images. Later, it was shown that the contrast between the LRNC and fibrous tissue increases after contrast injection $(36,55,84)$. The LRNC can be identified as a focal nonenhancing region on CE- $\mathrm{T}_{1} \mathrm{w}$ MR images (Figure 1). The LRNC area can be measured more reproducibly on the contrast-enhanced than the pre-contrast-enhanced images $(55,56)$. The coefficient of variation decreased from $33.5 \%$ to $17.6 \%$ for interreader measurements (85).

\section{FC status}

FC rupture or ulceration exposes the thrombogenic interior of the plaque to platelets and coagulation factors, which can lead to thrombus formation and distal embolization with clinical consequences. Hatsukami et al. (37) described for 
the first time that the FC status can be determined with MRI using a 3D-TOF sequence. High sensitivity (0.81) and specificity $(0.90)$ has been revealed for identifying the status of the $\mathrm{FC}$ by using multi-sequence (TOF, $\mathrm{T}_{1} \mathrm{w}$, proton density, and $\mathrm{T}_{2} \mathrm{w}$ ) MRI (39). Contrast-enhanced MRI (using a gadolinium-based contrast medium) enables to measure the dimensions of the FC (33). After gadolinium administration, the FC strongly enhances, whereas the LRNC enhances only slightly $(32,33,47)$. The interobserver agreement for assessment of $\mathrm{FC}$ status using preand post-contrast $\mathrm{T}_{1} \mathrm{w}$ TSE MRI is $\operatorname{good}(\kappa=0.64-0.74)(86)$.

\section{Ulceration}

Computed tomography angiography is considered the best noninvasive imaging modality to evaluate carotid plaque ulceration with a sensitivity and specificity of 94 and $99 \%$, respectively $(38,87,88)$. MRA can identify the presence of carotid ulcerations with a sensitivity similar to computed tomography angiography $(38,89)$. CE-MRA was superior (sensitivity: $82 \%$ ) to TOF-MRA (sensitivity: $55 \%$ ) for the detection of carotid ulcerations (38). In addition, CEMRA can identify ulcerations in calcified plaques, which is considered as a limitation of CTA (90). The addition of a longitudinal black blood MRA to a cross-sectional multi-sequence vessel wall MR imaging protocol increases the accuracy of detecting carotid atherosclerotic plaque ulcerations (91).

\section{Inflammation and neovascularization}

DCE-MRI can be used to quantify plaque microvasculature $(50,92) . \mathrm{K}^{\text {trans }}$ (volume transfer coefficient) as derived from pharmacokinetic modeling of DCE-MRI showed a significant correlation (with excised plaque neo-vascularity $(\mathrm{R}=0.41-0.7)(51,93,94)$. This technique has been shown to be highly reproducible and reliable (coefficient of variation: $16 \%$ for $\mathrm{K}^{\mathrm{trans}}$ ) (51). Alternatively, gadofosveset-enhanced MRI can be used to visualize plaque microvasculature without the need to use pharmacokinetic modeling. (95). van Hoof et al. reviewed the current status and future potential of DCE-MRI in the evaluation of plaque microvasculature (92). Recently, Yuan et al. demonstrated the feasibility of using a single sequence to acquire both high-resolution 4D CE-MRA and DCE-MRI to evaluate both plaque surface morphology and function (96).

Ultrasmall superparamagnetic particles of iron oxide (ferumoxtran-10)-enhanced MRI has been shown to be strongly associated with carotid plaque macrophage infiltration on histology as these particles are taken up by macrophages $(49,97,98)$. Ferumoxtran-10, however, is not broadly available. Alternatively, PET/MRI can be used to study plaque inflammation and composition with a single examination (99-101).

\section{Associations between plaque composition on MRI and stroke}

\section{Cross-sectional studies}

The ability of MRI to distinguish high-risk and lowrisk plaques was demonstrated in several proof-ofconcept studies. It has been demonstrated that enlarged plaque burden, IPH, LRNC, TRFC, inflammation and neovascularization are more common in symptomatic lesions as summarized below.

The relationship between carotid plaque burden and stroke risk has been demonstrated in various studies (Table 2). Carotid plaque burden was found to be greater in patients with recurrent stroke than that in those with firsttime stroke (114). Carotid plaque burden was significantly associated with ipsilateral acute cerebral infarction volume independent of the degree of carotid stenosis (115). Recently Liu et al. showed that carotid plaque burden in patients with $\geq 1.5 \mathrm{~mm}$ carotid plaques was associated with the presence of acute stroke (116). Based on numerous ultrasound studies, it is well known that plaque burden is a better parameter for risk prediction than measurement of the common carotid artery intima-media thickness (73).

It is well known that carotid IPH is associated with ipsilateral stroke in patients with $\geq 50 \%$ carotid stenosis $(46,115,117-120)$. IPH is more prevalent in the ipsilateral carotid artery compared with the contralateral, asymptomatic, side (60\% vs. 36\%) (102) (Table 2). Interestingly, in patients that were diagnosed with cryptogenic stroke and a nonstenotic $(<50 \%)$ carotid plaque, a higher prevalence of IPH was also reported by several studies on the ipsilateral side in several studies $(103,104,121,122)$, indicating that in a subgroup of these patients IPH may have been the underlying cause of the patients that were diagnosed with cryptogenic stroke. The presence of IPH also increased the risk for ipsilateral abnormalities on diffusion-weighted imaging (OR: 6.2, 95\% CI: 1.7-21.8, $\mathrm{P}<0.05)$ (123).

The need to assess the LRNC is justified by two important clinical needs: a large LRNC can lead to plaque rupture and LRNC size can be used to monitor treatment 
Table 2 Relation between carotid plaque MRI parameters and cerebrovascular symptoms

\begin{tabular}{|c|c|c|}
\hline $\begin{array}{l}\text { Plaque } \\
\text { component }\end{array}$ & Association with cerebrovascular symptoms & Predictive value for cerebrovascular events \\
\hline $\mathrm{IPH}$ & $\begin{array}{l}60 \% \text { symptomatic vs. } 36 \% \text { asymptomatic }(102) \\
37.5 \% \text { vs. } 0 \%(103,104) ;(\mathrm{HR}: 3.5 ; 95 \% \mathrm{Cl}: \\
1.05-11.87 ; \mathrm{P}=0.040)(105)\end{array}$ & $\begin{array}{l}\text { 5-6-fold higher risk for cerebrovascular events (HR: } 5.69 \text {; } 95 \% \mathrm{Cl} \text { : } \\
\text { 2.98-10.87) (74); (HR: 4.59, 95\% Cl: } 2.92-7.24)(46) \text {. IPH at baseline } \\
\text { predicts ipsilateral stroke in symptomatic (HR: } 10.2,95 \% \mathrm{Cl}: 4.6-22.5) \\
\text { and asymptomatic patients (HR: } 7.9,95 \% \mathrm{Cl}: 1.3-47.6)(6)\end{array}$ \\
\hline TRFC & $\begin{array}{l}\text { Patients with ruptured fibrous caps were } 23 \\
\text { times more likely to have had recent ischemic } \\
\text { neurological symptoms (95\% Cl: } 3-210)(107) \text {; } \\
\text { (HR: 5.756; } 95 \% \mathrm{Cl}: 1.9-17.3 ; \mathrm{P}=0.002)(105)\end{array}$ & $\begin{array}{l}\text { The hazard ratio for TRFC as predictor of stroke/TIA (HR: } 5.93 \text {, } \\
95 \% \mathrm{Cl} \text { : } 2.65-13.29)(46) \text {. The hazard ratio for TRFC as predictor of } \\
\text { cardiovascular events (HR: } 4.31 ; 95 \% \mathrm{Cl}: 1.67-11.1)(106)\end{array}$ \\
\hline Ulceration & $\begin{array}{l}86 \% \text { Symptomatic vs. } 36 \% \text { asymptomatic; } \\
P=0.039(109-113)\end{array}$ & \\
\hline
\end{tabular}

MRI, magnetic resonance imaging; IPH, intraplaque hemorrhage; LRNC, lipid-rich necrotic core; TRFC, thin or ruptured fibrous cap.

effects of lipid-lowering medication (124). The LRNC volume was shown to be significantly associated with the severity of cerebral infarction on DWI MRI (115). The multicenter CARE-II study of 687 symptomatic patients with an intima-media thickness $\geq 1.5 \mathrm{~mm}$ showed that the volume of the LRNC was significantly associated with TIA/ stroke $(116,125)$.

Several studies showed that there is a strong association between FC rupture on MRI and recent stroke or transit ischemic attack (TIA) $(105,107,126)$ (Table 2). In symptomatic patients, the volume of IPH was associated with FC disruption in carotid arteries (OR: 2.867, 95\% CI: $1.505-5.461 ; \mathrm{P}=0.001)$ after adjusting for clinical confounding factors and plaque burden (127). The irregular plaque surface as determined by MRI was an independent indicator for ipsilateral stroke in patients with carotid plaque ( $\geq 1.5 \mathrm{~mm})$ (128).

\section{Prospective longitudinal studies}

A large number of studies have reported the ability of MRI to identify patients at higher (or lower) risk of stroke $(6,46,74,105,129,130)$. Prevention of primary stroke in asymptomatic patients is very challenging since the degree of stenosis is not an adequate predictor for stroke $(131,132)$. Prospective studies in asymptomatic patients with carotid artery stenosis (50-79\%) showed that an increase in vessel wall thickness is associated with a larger risk for subsequent ischemic cerebrovascular symptoms (HR for $1 \mathrm{~mm}$ increase: 1.6; 95\% CI: 1.1-2.3) (129). The predictive value of IPH, LRNC, and TRFC $(46,74)$ as assessed by MRI for cerebrovascular events was determined in several studies. There were considerable differences between these studies regarding the degree of stenosis as well as the symptomatic status $(46,74)$. Meta-analyses showed a significant positive relationship between IPH and the risk of future ischemic events (HR: 5.69, 95\% CI: 2.98-10.87) (74), (HR: 4.59, 95\% CI: 2.92-7.24) (46). Over a median follow-up of 19.6 months, the presence of IPH was associated with a 6 -fold higher risk for cerebrovascular ischemic events (74) (Table 2). HRs for LRNC and TRFC as predictors of subsequent stroke/transient ischemic attack were (3.00, 95\% CI: $1.51-5.95)$ and $(5.93,95 \% \mathrm{CI}$ : 2.65-13.20), respectively (46). Lately, Schindler et al. performed a large meta-analysis of individual patient data from 7 cohort studies including 560 and 136 patients with symptomatic and asymptomatic carotid stenosis, respectively, with ipsilateral stroke as the primary endpoint (6). IPH was present in $51.6 \%$ and $29.4 \%$ of the patients with symptomatic and asymptomatic carotid stenosis, respectively. Presence of IPH at baseline increased the risk of ipsilateral stroke both in symptomatic (HR: 
10.2, 95\% CI: 4.6-22.5) and asymptomatic (HR: 7.9, 95\% CI: 1.3-47.6) patients (Table 2). A multivariate analysis in symptomatic patients showed that the risk of ipsilateral stroke was independently increased by the presence of IPH (HR: 11.0, 95\% CI: 4.8-25.1) and severe degree of stenosis (HR: 3.3, 95\% CI: 1.4-7.8) (6). Among symptomatic patients, carotid IPH was a stronger predictor of stroke risk than any known clinical risk factors. Among patients with symptomatic carotid stenosis, annualized event rates of ipsilateral stroke in those with IPH $v s$. those without were $9.0 \%$ vs. $0.7 \%$ (<50\% stenosis), $18.1 \%$ vs. $2.1 \%$ ( $50 \%$ to $69 \%$ stenosis), and $29.3 \%$ vs. $1.5 \%$ (70\% to $99 \%$ stenosis). This also confirms that also cryptogenic stroke patients with a non-stenotic ( $<50 \%$ stenosis) plaque with IPH are at increased risk for stroke. Annualized event rates among patients with asymptomatic carotid stenosis were $5.4 \% \mathrm{vs}$. $0.8 \%$ in those with and without IPH, respectively.

\section{Relation between plaque composition and clinical risk factors}

Noninvasive MRI can also be used to study the relation between plaque composition and clinical risk factors, also in lower risk populations, for which carotid endarterectomy specimens for histological studies are not available.

Carotid IPH is associated with clinical parameters that are known to affect stroke risk, i.e., positively with male sex, blood pressure, age and negatively with statin use (133-135). IPH and LRNC were more prevalent in men compared with women $(28.8 \%$ vs. $18.3 \%$ and $28.9 \%$ vs. $21.7 \%$, respectively) (136). In fact, for the same degree of carotid stenosis men have a larger LRNC than women (137). No association was found between total testosterone and LRNC in either sex. Higher total estradiol (OR: 1.58, 95\% CI: $1.03-2.40)$ and lower total testosterone (OR: 0.82, 95\% CI: 0.68-0.98) were associated with IPH in women but not in men (138).

In the Rotterdam study, a large population study of individuals with $\geq 2.5 \mathrm{~mm}$ plaque, it was shown that systolic blood pressure and pulse pressure were significantly positively associated with IPH (OR: $1.13,95 \%$ CI: 0.99-1.28; OR: $1.22,95 \%$ CI: $1.07-1.40$, respectively) after adjustment for age and sex (139). The serum insulin levels are also associated with IPH (OR: 1.42, 95\% CI: 1.12-1.7), while they were not associated with the presence of calcifications or a LRNC (140). Recently, Pletsch-Borba et al. (141) showed in a sub-analysis of 198 participants of the Rotterdam study, that hypertension is significantly associated with new IPH and new calcifications over a 4 year period (OR: 3.87, 95\% CI: 1.90-7.90; OR: 2.20, 95\% CI: 1.07-4.40, respectively), while higher levels of cholesterol were associated with LRNC progression (OR: 1.40, 95\% CI: $1.10-1.70)$.

Although statin therapy leads to a reduction in plaque progression (133), patients with IPH may be less sensitive to statin therapy (142). Moreover, statin treatment with any dosage was associated with a higher presence of calcifications (OR: 1.73, 95\% CI: 1.22-2.44), while a high dosage of statin treatment was also associated with a lower prevalence of LRNC (OR: 0.66, 95\% CI: 0.42-1.04) (143). In another large population study, the Multi-Ethnic Study of Atherosclerosis (MESA), of individuals with thickened carotid vessel wall $\geq 1 \mathrm{~mm}$, total plasma cholesterol, but not other established clinical risk factors, was strongly associated with the presence of LRNC on MRI.

\section{Determinants of plaque progression}

Progression of atherosclerosis is a complex phenomenon. Several studies have shown that IPH leads to enlargement of the lipid core, leading to plaque progression and destabilization $(142,144)$. Kwee et al. showed that the LRNC, IPH and FC status changed only in a minority of TIA/stroke patients with mild to moderate carotid stenosis over a one year period (145).

\section{Ability of MRI to measure treatment effects}

High resolution MRI studies on the effects of different statin therapies and different dosages on plaque composition were summarized in a systematic review and meta-analysis (124). Although there was no significant difference in LRNC size at 1-6 months and at 7-12 months following initiation of statin therapy, at $>12$ months, there was a significant decrease in LRNC volume.

In the ORION trial, the mean percentage of the vessel wall composed of LRNC on MRI decreased by $>40 \%$ $(\mathrm{P}=0.005)$ in both low and high dose of rosuvastatin therapy (146). T2-mapping demonstrated a depleted lipid content of the carotid plaque after 3 months of highintensity statin treatment (atorvastatin $80 \mathrm{mg}$ ) from $10.3 \%$ (7.2-14.2) to $7.4 \%$ (5.4-10.0), $\mathrm{P}=0.002$ (147).

\section{Clinical perspectives and future outlook}

The continued high incidence of stroke despite advances 
in optimized medical therapy strongly indicates that individual response to therapy may vary widely. Numerous histopathological studies performed on CEA specimens have shown that specific plaque features are associated with an increased stroke incidence $(26,27)$. Highresolution, multi-contrast carotid MRI can identify and quantify atherosclerotic components within carotid plaques $(7,10,34,63)$. Multi-contrast sequences enable to obtain images with different contrast with a short single sequence $(30,62)$. MRI is currently recognized as the most valuable imaging modality for carotid plaque burden quantification and non-invasive assessment of plaque composition $(7,8)$. Furthermore, MRI is well validated, highly reproducible and can be used to predict stroke and evaluate treatment effects $(8,10,36)$. Carotid MRI has increased our knowledge on carotid atherosclerotic disease, since MRI studies allowed, for the first time, to follow changes in plaque composition over time, also before clinical symptoms occur $(35,148-150)$. It provides an excellent opportunity of improved stroke risk assessment, noninvasive monitoring of disease progression and evaluation of therapeutic efficacy $(6,46,74,118,129)$.

MRI may be used to identify a subgroup of patients that may benefit from expensive new treatments, such as antiinflammatory therapy or intensified lipid-lowering therapy. IPH on MRI is currently the most promising approach for implementation in daily clinical practice in the near future, since IPH is easy to recognize on MR images, it can be visualized with a standard MRI sequence with a scan time of a few minutes using a standard neurovascular coil (45). A recent meta-analysis showed that IPH is a strong independent predictor for stroke in symptomatic as well as asymptomatic patients with carotid stenosis, independent of degree of stenosis and stronger than any known clinical risk factors (6).

In asymptomatic patients with a significant carotid artery stenosis performance of carotid endarterectomy is highly controversial (151). The number needed to treat to prevent one stroke within 5 years in this asymptomatic population is very high and annual stroke rates due to best medical treatment may be declining (152-154). Therefore, the recent guidelines of the European Society for Vascular Surgery (ESVS) state that, while waiting for results of large clinical trials and the development of validated algorithms for patient selection, the presence of one or more imaging features, such as large plaque burden of intraplaque haemorrhage on MRI may be useful to select higher risk for stroke patients (155). In these clinical guidelines, the advantage of MRA and CTA compared to Duplex ultrasound to visualise simultaneously the aortic arch, supra-aortic trunks, carotid bifurcation, distal internal carotid artery, and the intracranial circulation is also highlighted (155). They state that such an examination is mandatory if a patient is considered for carotid artery stenting (155). A short additional MRI sequence of a few minutes to quantify plaque burden and to identify intraplaque haemorrhage can be easily added to an MRA examination.

IPH and LRNC are observed not only in patients with significant stenosis, but also in patients with non-significant $(<50 \%)$ stenosis $(103,104,121,122)$. These features have been acknowledged as critical factors for clinical disease assessment (46). IPH has been linked with accelerated plaque progression, luminal narrowing, and clinical events $(142,144)$. The LRNC volume can be used to monitor therapeutic effects of lipid lowering treatment (124). Carotid plaque MRI can also be helpful in borderline clinical cases. Expert consensus recommendations on carotid vessel wall MRI have recently been published (63). In this white paper detailed recommendations on MRI protocols are provided. For identification of the LRNC and the FC status, currently contrast injection is still preferred $(36,55,85)$. Recently, fast three-dimensional sequences have been developed with a large longitudinal coverage $(52,53,156)$. Artificial intelligence may lead to further improvements in MR image quality, shorter scan times and it can provide tools for automated image analysis (157). The development and validation of risk prediction models that include carotid plaque MRI findings can further aid in improved risk stratification.

Large clinical trials are warranted to study whether carotid plaque MRI can be used to select patients that benefit from carotid revascularization, but also to identify patients that can be safely treated with best medical treatment alone.

Further studies might be needed to investigate the relation between MRI features and the most suitable type of therapy. The introduction of hybrid PET/MRI systems allows for the combination of anatomical imaging with MRI, and metabolic/functional imaging with PET (101). Together these advances are expected to lead to a reduction in stroke and the substantial economic burden associated with stroke mortality, morbidity and long-term disability.

\section{Acknowledgments}

Funding: None. 


\section{Footnote}

Provenance and Peer Review: This article was commissioned by the Guest Editor (Dr. Kosmas I. Paraskevas) for the series "Carotid Artery Stenosis and Stroke: Prevention and Treatment Part I" published in Annals of Translational Medicine. The article was sent for external peer review organized by the Guest Editor and the editorial office.

Conflicts of Interest: All authors have completed the ICMJE uniform disclosure form (available at http://dx.doi. org/10.21037/atm-2020-cass-16). The series "Carotid Artery Stenosis and Stroke: Prevention and Treatment Part I" was commissioned by the editorial office without any funding or sponsorship. MK reports grants from Netherlands Organization for Scientific Research, during the conduct of the study. AF reports grants from European Union's Horizon 2020 research and innovation programme under the Marie Sklodowska-Curie grant agreement No 722609, outside the submitted work. MEK reports grants from Netherlands Organization for Scientific Research, grants from European Union's Horizon 2020, grants from Netherlands Organization for Scientific Research, during the conduct of the study. The other authors have no other conflicts of interest to declare.

Ethical Statement: The authors are accountable for all aspects of the work in ensuring that questions related to the accuracy or integrity of any part of the work are appropriately investigated and resolved.

Open Access Statement: This is an Open Access article distributed in accordance with the Creative Commons Attribution-NonCommercial-NoDerivs 4.0 International License (CC BY-NC-ND 4.0), which permits the noncommercial replication and distribution of the article with the strict proviso that no changes or edits are made and the original work is properly cited (including links to both the formal publication through the relevant DOI and the license). See: https://creativecommons.org/licenses/by-nc-nd/4.0/.

\section{References}

1. Lusis AJ. Atherosclerosis. Nature 2000;407:233-41.

2. Ross R. Atherosclerosis - An Inflammatory Disease. N Engl J Med 1999;340:115-26.

3. Gijsen FJ, Wentzel JJ, Thury A, et al. Strain distribution over plaques in human coronary arteries relates to shear stress. Am J Physiol Heart Circ Physiol 2008;295:H1608-14.

4. Slager CJ, Wentzel JJ, Gijsen FJ, et al. The role of shear stress in the generation of rupture-prone vulnerable plaques. Nat Clin Pract Cardiovasc Med 2005;2:401-7.

5. Vengrenyuk Y, Carlier S, Xanthos S, et al. A hypothesis for vulnerable plaque rupture due to stress-induced debonding around cellular microcalcifications in thin fibrous caps. Proc Natl Acad Sci U S A 2006;103:14678-83.

6. Schindler A, Schinner R, Altaf N, et al. Prediction of Stroke Risk by Detection of Hemorrhage in Carotid Plaques: Meta-Analysis of Individual Patient Data. JACC Cardiovasc Imaging 2020;13:395-406.

7. Cappendijk VC, Cleutjens KB, Kessels AG, et al. Assessment of human atherosclerotic carotid plaque components with multisequence MR imaging: initial experience. Radiology 2005;234:487-92.

8. den Hartog AG, Bovens SM, Koning W, et al. Current status of clinical magnetic resonance imaging for plaque characterisation in patients with carotid artery stenosis. Eur J Vasc Endovasc Surg 2013;45:7-21.

9. Saam T, Kerwin WS, Chu B, et al. Sample size calculation for clinical trials using magnetic resonance imaging for the quantitative assessment of carotid atherosclerosis. J Cardiovasc Magn Reson 2005;7:799-808.

10. Yuan C, Kerwin WS, Yarnykh VL, et al. MRI of atherosclerosis in clinical trials. NMR Biomed 2006;19:636-54.

11. Falk E, Shah PK, Fuster V. Coronary plaque disruption. Circulation 1995;92:657-71.

12. Virmani R, Kolodgie FD, Burke AP, et al. Lessons from sudden coronary death: a comprehensive morphological classification scheme for atherosclerotic lesions. Arterioscler Thromb Vasc Biol 2000;20:1262-75.

13. Falk E. Stable versus unstable atherosclerosis: clinical aspects. Am Heart J 1999;138:S421-5.

14. Virmani R, Ladich ER, Burke AP, et al. Histopathology of carotid atherosclerotic disease. Neurosurgery 2006;59:S219-27; discussion S3-13.

15. Bentzon JF, Otsuka F, Virmani R, et al. Mechanisms of plaque formation and rupture. Circ Res 2014;114:1852-66.

16. Tabas I, Bornfeldt KE. Macrophage Phenotype and Function in Different Stages of Atherosclerosis. Circ Res 2016;118:653-67.

17. Murray PJ, Allen JE, Biswas SK, et al. Macrophage activation and polarization: nomenclature and experimental guidelines. Immunity 2014;41:14-20.

18. Libby P. Inflammation in atherosclerosis. Nature 
2002;420:868-74.

19. Gibson MS, Domingues N, Vieira OV. Lipid and Nonlipid Factors Affecting Macrophage Dysfunction and Inflammation in Atherosclerosis. Front Physiol 2018;9:654.

20. Sluimer JC, Daemen MJ. Novel concepts in atherogenesis: angiogenesis and hypoxia in atherosclerosis. J Pathol 2009;218:7-29.

21. Imparato AM, Riles TS, Gorstein F. The carotid bifurcation plaque: pathologic findings associated with cerebral ischemia. Stroke 1979;10:238-45.

22. Persson AV, Robichaux WT, Silverman M. The natural history of carotid plaque development. Arch Surg 1983;118:1048-52.

23. Bornstein NM, Krajewski A, Lewis AJ, et al. Clinical significance of carotid plaque hemorrhage. Arch Neurol 1990;47:958-9.

24. Kolodgie FD, Gold HK, Burke AP, et al. Intraplaque hemorrhage and progression of coronary atheroma. $\mathrm{N}$ Engl J Med 2003;349:2316-25.

25. Groen HC, Gijsen FJ, van der Lugt A, et al. Plaque rupture in the carotid artery is localized at the high shear stress region: a case report. Stroke 2007;38:2379-81.

26. Hellings WE, Peeters W, Moll FL, et al. Composition of carotid atherosclerotic plaque is associated with cardiovascular outcome: a prognostic study. Circulation 2010;121:1941-50.

27. Fleiner M, Kummer M, Mirlacher M, et al. Arterial neovascularization and inflammation in vulnerable patients: early and late signs of symptomatic atherosclerosis. Circulation 2004;110:2843-50.

28. Kwee RM, van Oostenbrugge RJ, Hofstra L, et al. Identifying vulnerable carotid plaques by noninvasive imaging. Neurology 2008;70:2401-9.

29. Moody AR, Murphy RE, Morgan PS, et al. Characterization of complicated carotid plaque with magnetic resonance direct thrombus imaging in patients with cerebral ischemia. Circulation 2003;107:3047-52.

30. Wang J, Bornert P, Zhao H, et al. Simultaneous noncontrast angiography and intraplaque hemorrhage (SNAP) imaging for carotid atherosclerotic disease evaluation. Magn Reson Med 2013;69:337-45.

31. Zhou T, Jia S, Wang X, et al. Diagnostic performance of MRI for detecting intraplaque hemorrhage in the carotid arteries: a meta-analysis. Eur Radiol 2019;29:5129-38.

32. Yuan C, Kerwin WS, Ferguson MS, et al. Contrastenhanced high resolution MRI for atherosclerotic carotid artery tissue characterization. J Magn Reson Imaging 2002;15:62-7.
33. Cai J, Hatsukami TS, Ferguson MS, et al. In vivo quantitative measurement of intact fibrous cap and lipidrich necrotic core size in atherosclerotic carotid plaque: comparison of high-resolution, contrast-enhanced magnetic resonance imaging and histology. Circulation 2005;112:3437-44.

34. Yuan C, Mitsumori LM, Ferguson MS, et al. In vivo accuracy of multispectral magnetic resonance imaging for identifying lipid-rich necrotic cores and intraplaque hemorrhage in advanced human carotid plaques. Circulation 2001;104:2051-6.

35. Cai JM, Hatsukami TS, Ferguson MS, et al. Classification of human carotid atherosclerotic lesions with in vivo multicontrast magnetic resonance imaging. Circulation 2002;106:1368-73.

36. Saam T, Ferguson MS, Yarnykh VL, et al. Quantitative evaluation of carotid plaque composition by in vivo MRI. Arterioscler Thromb Vasc Biol 2005;25:234-9.

37. Hatsukami TS, Ross R, Polissar NL, et al. Visualization of fibrous cap thickness and rupture in human atherosclerotic carotid plaque in vivo with high-resolution magnetic resonance imaging. Circulation 2000;102:959-64.

38. Etesami M, Hoi Y, Steinman DA, et al. Comparison of carotid plaque ulcer detection using contrast-enhanced and time-of-flight MRA techniques. AJNR Am J Neuroradiol 2013;34:177-84.

39. Mitsumori LM, Hatsukami TS, Ferguson MS, et al. In vivo accuracy of multisequence $M R$ imaging for identifying unstable fibrous caps in advanced human carotid plaques. J Magn Reson Imaging 2003;17:410-20.

40. Chen S, Zhao H, Li J, et al. Evaluation of carotid atherosclerotic plaque surface characteristics utilizing simultaneous noncontrast angiography and intraplaque hemorrhage (SNAP) technique. J Magn Reson Imaging 2018;47:634-9.

41. Underhill HR, Hatsukami TS, Fayad ZA, et al. MRI of carotid atherosclerosis: clinical implications and future directions. Nat Rev Cardiol 2010;7:165-73.

42. Toussaint JF, LaMuraglia GM, Southern JF, et al. Magnetic resonance images lipid, fibrous, calcified, hemorrhagic, and thrombotic components of human atherosclerosis in vivo. Circulation 1996;94:932-8.

43. von Ingersleben G, Schmiedl UP, Hatsukami TS, et al. Characterization of atherosclerotic plaques at the carotid bifurcation: correlation of high-resolution MR imaging with histologic analysis--preliminary study. Radiographics 1997;17:1417-23.

44. Franceschini N, Giambartolomei C, de Vries PS, et al. 
GWAS and colocalization analyses implicate carotid intima-media thickness and carotid plaque loci in cardiovascular outcomes. Nat Commun 2018;9:5141.

45. van der Kolk AG, de Borst GJ, den Hartog AG, et al. Hyperintense carotid plaque on $\mathrm{T}(1)$-weighted turbofield echo MRI in symptomatic patients with low-grade carotid stenosis and carotid occlusion. Cerebrovasc Dis 2010;30:221-9.

46. Gupta A, Baradaran H, Schweitzer AD, et al. Carotid plaque MRI and stroke risk: a systematic review and metaanalysis. Stroke 2013;44:3071-7.

47. Wasserman BA, Smith WI, Trout HH 3rd, et al. Carotid artery atherosclerosis: in vivo morphologic characterization with gadolinium-enhanced double-oblique MR imaging initial results. Radiology 2002;223:566-73.

48. Trivedi R, J UKI, Gillard J. Accumulation of ultrasmall superparamagnetic particles of iron oxide in human atherosclerotic plaque. Circulation 2003;108:e140; author reply e140.

49. Kooi ME, Cappendijk VC, Cleutjens KB, et al. Accumulation of ultrasmall superparamagnetic particles of iron oxide in human atherosclerotic plaques can be detected by in vivo magnetic resonance imaging. Circulation 2003;107:2453-8.

50. Kerwin W, Hooker A, Spilker M, et al. Quantitative magnetic resonance imaging analysis of neovasculature volume in carotid atherosclerotic plaque. Circulation 2003;107:851-6.

51. Gaens ME, Backes W, Rozel S, et al. Dynamic contrastenhanced MR imaging of carotid atherosclerotic plaque: model selection, reproducibility, and validation. Radiology 2013;266:271-9.

52. Yarnykh VL, Yuan C. T1-insensitive flow suppression using quadruple inversion-recovery. Magn Reson Med 2002;48:899-905.

53. Li L, Chai JT, Biasiolli L, et al. Black-blood multicontrast imaging of carotid arteries with DANTE-prepared 2D and 3D MR imaging. Radiology 2014;273:560-9.

54. Chu B, Kampschulte A, Ferguson MS, et al. Hemorrhage in the atherosclerotic carotid plaque: a high-resolution MRI study. Stroke 2004;35:1079-84.

55. Takaya N, Cai J, Ferguson MS, et al. Intra- and interreader reproducibility of magnetic resonance imaging for quantifying the lipid-rich necrotic core is improved with gadolinium contrast enhancement. J Magn Reson Imaging 2006;24:203-10.

56. Saam T, Hatsukami TS, Yarnykh VL, et al. Reader and platform reproducibility for quantitative assessment of carotid atherosclerotic plaque using 1.5T Siemens, Philips, and General Electric scanners. J Magn Reson Imaging 2007;26:344-52.

57. Koktzoglou I, Chung YC, Mani V, et al. Multislice darkblood carotid artery wall imaging: a $1.5 \mathrm{~T}$ and $3.0 \mathrm{~T}$ comparison. J Magn Reson Imaging 2006;23:699-705.

58. Vidal A, Bureau Y, Wade T, et al. Scan-rescan and intraobserver variability of magnetic resonance imaging of carotid atherosclerosis at $1.5 \mathrm{~T}$ and $3.0 \mathrm{~T}$. Phys Med Biol 2008;53:6821-35.

59. Saam T, Raya JG, Cyran CC, et al. High resolution carotid black-blood 3T MR with parallel imaging and dedicated 4-channel surface coils. Journal of Cardiovascular Magnetic Resonance 2009;11:41.

60. Balu N, Yarnykh VL, Scholnick J, et al. Improvements in carotid plaque imaging using a new eight-element phased array coil at 3T. J Magn Reson Imaging 2009;30:1209-14.

61. Hadley JR, Roberts JA, Goodrich KC, et al. Relative RF coil performance in carotid imaging. Magn Reson Imaging 2005;23:629-39.

62. Fan Z, Yu W, Xie Y, et al. Multi-contrast atherosclerosis characterization (MATCH) of carotid plaque with a single 5 -min scan: technical development and clinical feasibility. J Cardiovasc Magn Reson 2014;16:53.

63. Saba L, Yuan C, Hatsukami TS, et al. Carotid Artery Wall Imaging: Perspective and Guidelines from the ASNR Vessel Wall Imaging Study Group and Expert Consensus Recommendations of the American Society of Neuroradiology. AJNR Am J Neuroradiol 2018;39:E9-31.

64. Glagov S, Weisenberg E, Zarins CK, et al. Compensatory enlargement of human atherosclerotic coronary arteries. N Engl J Med 1987;316:1371-5.

65. Varghese A, Crowe LA, Mohiaddin RH, et al. Inter-study reproducibility of $3 \mathrm{D}$ volume selective fast spin echo sequence for quantifying carotid artery wall volume in asymptomatic subjects. Atherosclerosis 2005;183:361-6.

66. Zhu C, Teng Z, Sadat U, et al. Normalized wall index specific and MRI-based stress analysis of atherosclerotic carotid plaques: a study comparing acutely symptomatic and asymptomatic patients. Circ J 2010;74:2360-4.

67. Kerwin W, Xu D, Liu F, et al. Magnetic resonance imaging of carotid atherosclerosis: plaque analysis. Top Magn Reson Imaging 2007;18:371-8.

68. Li F, Yarnykh VL, Hatsukami TS, et al. Scan-rescan reproducibility of carotid atherosclerotic plaque morphology and tissue composition measurements using multicontrast MRI at 3T. J Magn Reson Imaging 2010;31:168-76. 
69. Wang J, Yarnykh VL, Hatsukami T, et al. Improved suppression of plaque-mimicking artifacts in black-blood carotid atherosclerosis imaging using a multislice motionsensitized driven-equilibrium (MSDE) turbo spin-echo (TSE) sequence. Magn Reson Med 2007;58:973-81.

70. Luo Y, Polissar N, Han C, et al. Accuracy and uniqueness of three in vivo measurements of atherosclerotic carotid plaque morphology with black blood MRI. Magn Reson Med 2003;50:75-82

71. Balu N, Yarnykh VL, Chu B, et al. Carotid plaque assessment using fast 3D isotropic resolution black-blood MRI. Magn Reson Med 2011;65:627-37.

72. Duivenvoorden R, de Groot E, Elsen BM, et al. In vivo quantification of carotid artery wall dimensions: 3.0-Tesla MRI versus B-mode ultrasound imaging. Circ Cardiovasc Imaging 2009;2:235-42.

73. Paraskevas KI, Sillesen HH, Rundek T, et al. Carotid Intima-Media Thickness Versus Carotid Plaque Burden for Predicting Cardiovascular Risk. Angiology 2020;71:108-11.

74. Saam T, Hetterich H, Hoffmann V, et al. Meta-Analysis and Systematic Review of the Predictive Value of Carotid Plaque Hemorrhage on Cerebrovascular Events by Magnetic Resonance Imaging. J Am Coll Cardiol 2013;62:1081-91.

75. den Hartog AG, Bovens S, Koning W, et al. Current status of clinical magnetic resonance imaging for plaque characterisation in patients with carotid artery stenosis. Eur J Vasc Endovasc Surg 2013;45:7-21.

76. Cappendijk VC, Cleutjens KB, Heeneman S, et al. In vivo detection of hemorrhage in human atherosclerotic plaques with magnetic resonance imaging. J Magn Reson Imaging 2004;20:105-10.

77. Ota H, Yarnykh VL, Ferguson MS, et al. Carotid intraplaque hemorrhage imaging at 3.0-T MR imaging: comparison of the diagnostic performance of three T1weighted sequences. Radiology 2010;254:551-63.

78. Liu J, Sun J, Balu N, et al. Semiautomatic carotid intraplaque hemorrhage volume measurement using 3D carotid MRI. J Magn Reson Imaging 2019;50:1055-62.

79. Hofman JM, Branderhorst WJ, ten Eikelder HM, et al. Quantification of atherosclerotic plaque components using in vivo MRI and supervised classifiers. Magn Reson Med 2006;55:790-9.

80. Qiao Y, Etesami M, Malhotra S, et al. Identification of intraplaque hemorrhage on MR angiography images: a comparison of contrast-enhanced mask and time-of-flight techniques. AJNR Am J Neuroradiol 2011;32:454-9.

81. Coolen BF, Poot DH, Liem MI, et al. Three-dimensional quantitative T1 and T2 mapping of the carotid artery: Sequence design and in vivo feasibility. Magn Reson Med 2016;75:1008-17.

82. Qi H, Sun J, Qiao H, et al. Carotid Intraplaque Hemorrhage Imaging with Quantitative Vessel Wall T1 Mapping: Technical Development and Initial Experience. Radiology 2018;287:276-84.

83. Toussaint JF, Southern JF, Fuster V, et al. T2weighted contrast for NMR characterization of human atherosclerosis. Arterioscler Thromb Vasc Biol 1995;15:1533-42.

84. Cappendijk VC, Kessels AG, Heeneman S, et al. Comparison of lipid-rich necrotic core size in symptomatic and asymptomatic carotid atherosclerotic plaque: Initial results. J Magn Reson Imaging 2008;27:1356-61.

85. Young VE, Patterson AJ, Sadat U, et al. Diffusionweighted magnetic resonance imaging for the detection of lipid-rich necrotic core in carotid atheroma in vivo. Neuroradiology 2010;52:929-36.

86. Kwee RM, van Engelshoven JM, Mess WH, et al. Reproducibility of fibrous cap status assessment of carotid artery plaques by contrast-enhanced MRI. Stroke 2009;40:3017-21.

87. Saba L, Caddeo G, Sanfilippo R, et al. Efficacy and sensitivity of axial scans and different reconstruction methods in the study of the ulcerated carotid plaque using multidetector-row CT angiography: comparison with surgical results. AJNR Am J Neuroradiol 2007;28:716-23.

88. Cumming MJ, Morrow IM. Carotid artery stenosis: a prospective comparison of CT angiography and conventional angiography. AJR Am J Roentgenol 1994;163:517-23.

89. Brinjikji W, Huston J, 3rd, Rabinstein AA, et al. Contemporary carotid imaging: from degree of stenosis to plaque vulnerability. J Neurosurg 2016;124:27-42.

90. Yuan J, Usman A, Das T, et al. Imaging Carotid Atherosclerosis Plaque Ulceration: Comparison of Advanced Imaging Modalities and Recent Developments. Am J Neuroradiol 2017;38:664-71.

91. Yu W, Underhill HR, Ferguson MS, et al. The added value of longitudinal black-blood cardiovascular magnetic resonance angiography in the cross sectional identification of carotid atherosclerotic ulceration. J Cardiovasc Magn Reson 2009;11:31.

92. van Hoof RH, Heeneman S, Wildberger JE, et al. Dynamic Contrast-Enhanced MRI to Study Atherosclerotic Plaque Microvasculature. Curr Atheroscler Rep 2016;18:33.

93. Kerwin WS, Oikawa M, Yuan C, et al. MR imaging of 
adventitial vasa vasorum in carotid atherosclerosis. Magn Reson Med 2008;59:507-14.

94. van Hoof RHM, Voo SA, Sluimer JC, et al. Vessel wall and adventitial DCE-MRI parameters demonstrate similar correlations with carotid plaque microvasculature on histology. J Magn Reson Imaging 2017;46:1053-9.

95. Lobbes MB, Heeneman S, Passos VL, et al. Gadofosvesetenhanced magnetic resonance imaging of human carotid atherosclerotic plaques: a proof-of-concept study. Invest Radiol 2010;45:275-81.

96. Yuan J, Makris G, Patterson A, et al. Relationship between carotid plaque surface morphology and perfusion: a 3D DCE-MRI study. MAGMA 2018;31:191-9.

97. Trivedi RA, Mallawarachi C, JM UK-I, et al. Identifying inflamed carotid plaques using in vivo USPIO-enhanced MR imaging to label plaque macrophages. Arterioscler Thromb Vasc Biol 2006;26:1601-6.

98. Chan JM, Monaco C, Wylezinska-Arridge M, et al. Imaging of the vulnerable carotid plaque: biological targeting of inflammation in atherosclerosis using iron oxide particles and MRI. Eur J Vasc Endovasc Surg 2014;47:462-9.

99. Bachi K, Mani V, Kaufman AE, et al. Imaging plaque inflammation in asymptomatic cocaine addicted individuals with simultaneous positron emission tomography/magnetic resonance imaging. World J Radiol 2019;11:62-73.

100.Li X, Heber D, Leike T, et al. [68Ga]Pentixafor-PET/ MRI for the detection of Chemokine receptor 4 expression in atherosclerotic plaques. Eur J Nucl Med Mol Imaging 2018;45:558-66.

101.Aizaz M, Moonen R, Pol J, et al. PET/MR imaging of atherosclerosis. Cardiovasc Diagn Ther 2020;10:1120-39.

102. Murphy RE, Moody AR, Morgan PS, et al. Prevalence of complicated carotid atheroma as detected by magnetic resonance direct thrombus imaging in patients with suspected carotid artery stenosis and previous acute cerebral ischemia. Circulation 2003;107:3053-8.

103. Freilinger TM, Schindler A, Schmidt C, et al. Prevalence of nonstenosing, complicated atherosclerotic plaques in cryptogenic stroke. JACC Cardiovasc Imaging 2012;5:397-405.

104.Hyafil F, Schindler A, Sepp D, et al. High-risk plaque features can be detected in non-stenotic carotid plaques of patients with ischaemic stroke classified as cryptogenic using combined (18)F-FDG PET/MR imaging. Eur J Nucl Med Mol Imaging 2016;43:270-9.

105. Kwee RM, van Oostenbrugge RJ, Mess WH, et al. MRI of carotid atherosclerosis to identify TIA and stroke patients who are at risk of a recurrence. J Magn Reson Imaging 2013;37:1189-94.

106. Sun J, Zhao XQ, Balu N, et al. Carotid Plaque Lipid Content and Fibrous Cap Status Predict Systemic CV Outcomes: The MRI Substudy in AIM-HIGH. JACC Cardiovasc Imaging 2017;10:241-9.

107. Yuan C, Zhang S-x, Polissar NL, et al. Identification of fibrous cap rupture with magnetic resonance imaging is highly associated with recent transient ischemic attack or stroke. Circulation 2002;105:181-5.

108. Nandalur KR, Hardie AD, Raghavan P, et al. Composition of the stable carotid plaque: insights from a multidetector computed tomography study of plaque volume. Stroke 2007;38:935-40.

109. Demarco JK, Ota H, Underhill HR, et al. MR carotid plaque imaging and contrast-enhanced MR angiography identifies lesions associated with recent ipsilateral thromboembolic symptoms: an in vivo study at 3T. AJNR Am J Neuroradiol 2010;31:1395-402.

110. Sitzer M, Muller W, Siebler M, et al. Plaque ulceration and lumen thrombus are the main sources of cerebral microemboli in high-grade internal carotid artery stenosis. Stroke 1995;26:1231-3.

111. Troyer A, Saloner D, Pan XM, et al. Major carotid plaque surface irregularities correlate with neurologic symptoms. J Vasc Surg 2002;35:741-7.

112. Eliasziw M, Streifler JY, Fox AJ, et al. Significance of plaque ulceration in symptomatic patients with high-grade carotid stenosis. North American Symptomatic Carotid Endarterectomy Trial. Stroke 1994;25:304-8.

113. Rothwell PM, Gibson R, Warlow CP. Interrelation Between Plaque Surface Morphology and Degree of Stenosis on Carotid Angiograms and the Risk of Ischemic Stroke in Patients With Symptomatic Carotid Stenosis. Stroke 2000;31:615-21.

114. Liu XS, Zhao HL, Cao Y, et al. Comparison of Carotid Atherosclerotic Plaque Characteristics by High-Resolution Black-Blood MR Imaging between Patients with FirstTime and Recurrent Acute Ischemic Stroke. American Journal of Neuroradiology 2012;33:1257-61.

115.Zhao H, Zhao X, Liu X, et al. Association of carotid atherosclerotic plaque features with acute ischemic stroke: A magnetic resonance imaging study. Eur J Radiol 2013;82:e465-70.

116. Liu Y, Wang M, Zhang B, et al. Size of carotid artery intraplaque hemorrhage and acute ischemic stroke: a cardiovascular magnetic resonance Chinese atherosclerosis risk evaluation study. J Cardiovasc Magn Reson 
2019;21:36.

117. Gao P, Chen ZQ, Bao YH, et al. Correlation between carotid intraplaque hemorrhage and clinical symptoms: systematic review of observational studies. Stroke 2007;38:2382-90.

118. Altaf N, Daniels L, Morgan PS, et al. Detection of intraplaque hemorrhage by magnetic resonance imaging in symptomatic patients with mild to moderate carotid stenosis predicts recurrent neurological events. J Vasc Surg 2008;47:337-42.

119. Turc G, Oppenheim C, Naggara O, et al. Relationships between recent intraplaque hemorrhage and stroke risk factors in patients with carotid stenosis: the HIRISC study. Arterioscler Thromb Vasc Biol 2012;32:492-9.

120. Seyedsaadat SM, Rizvi A, Alzuabi M, et al. Correlation of MRI-detected vulnerable carotid plaques with clinical presentation: a systematic review and meta-analysis. J Neurosurg Sci 2020;64:263-71.

121. Singh N, Moody AR, Panzov V, et al. Carotid Intraplaque Hemorrhage in Patients with Embolic Stroke of Undetermined Source. J Stroke Cerebrovasc Dis 2018;27:1956-9.

122. Gupta A, Gialdini G, Lerario MP, et al. Magnetic resonance angiography detection of abnormal carotid artery plaque in patients with cryptogenic stroke. J Am Heart Assoc 2015;4:e02012.

123. Altaf N, Goode SD, Beech A, et al. Plaque hemorrhage is a marker of thromboembolic activity in patients with symptomatic carotid disease. Radiology 2011;258:538-45.

124.Brinjikji W, Lehman VT, Kallmes DF, et al. The effects of statin therapy on carotid plaque composition and volume: A systematic review and meta-analysis. J Neuroradiol 2017;44:234-40.

125.Zhao X, Li R, Hippe DS, et al. Chinese Atherosclerosis Risk Evaluation (CARE II) study: a novel crosssectional, multicentre study of the prevalence of high-risk atherosclerotic carotid plaque in Chinese patients with ischaemic cerebrovascular events-design and rationale. Stroke Vasc Neurol 2017;2:15-20.

126. Saam T, Cai J, Ma L, et al. Comparison of symptomatic and asymptomatic atherosclerotic carotid plaque features with in vivo MR imaging. Radiology 2006;240:464-72.

127.Cui Y, Qiao H, Ma L, et al. Association of Age and Size of Carotid Artery Intraplaque Hemorrhage and Minor Fibrous Cap Disruption: A High Resolution Magnetic Resonance Imaging Study. J Atheroscler Thromb 2018;25:1222-30.

128.Zhou D, Li J, Liu D, et al. Irregular surface of carotid atherosclerotic plaque is associated with ischemic stroke: a magnetic resonance imaging study. J Geriatr Cardiol 2019;16:872-9.

129. Takaya N, Yuan C, Chu B, et al. Association between carotid plaque characteristics and subsequent ischemic cerebrovascular events: a prospective assessment with MRI--initial results. Stroke 2006;37:818-23.

130. Hosseini AA, Kandiyil N, Macsweeney ST, et al. Carotid plaque hemorrhage on magnetic resonance imaging strongly predicts recurrent ischemia and stroke. Ann Neurol 2013;73:774-84.

131. Cai Y, He L, Yuan C, et al. Atherosclerotic plaque features and distribution in bilateral carotid arteries of asymptomatic elderly population: A 3D multicontrast MR vessel wall imaging study. Eur J Radiol 2017;96:6-11.

132.Xu D, Hippe DS, Underhill HR, et al. Prediction of highrisk plaque development and plaque progression with the carotid atherosclerosis score. JACC Cardiovasc Imaging 2014;7:366-73.

133. Kwee RM, van Oostenbrugge RJ, Prins MH, et al. Symptomatic patients with mild and moderate carotid stenosis: plaque features at MRI and association with cardiovascular risk factors and statin use. Stroke 2010;41:1389-93.

134. Cheung HM, Moody AR, Singh N, et al. Late stage complicated atheroma in low-grade stenotic carotid disease: MR imaging depiction--prevalence and risk factors. Radiology 2011;260:841-7.

135. Singh N, Moody AR, Zhang B, et al. Age-Specific Sex Differences in Magnetic Resonance Imaging-Depicted Carotid Intraplaque Hemorrhage. Stroke 2017;48:2129-35.

136.van den Bouwhuijsen QJ, Vernooij MW, Hofman A, et al. Determinants of magnetic resonance imaging detected carotid plaque components: the Rotterdam Study. Eur Heart J 2012;33:221-9.

137. Ota H, Reeves MJ, Zhu DC, et al. Sex differences in patients with asymptomatic carotid atherosclerotic plaque: in vivo 3.0-T magnetic resonance study. Stroke 2010;41:1630-5.

138. Glisic M, Mujaj B, Rueda-Ochoa OL, et al. Associations of Endogenous Estradiol and Testosterone Levels With Plaque Composition and Risk of Stroke in Subjects With Carotid Atherosclerosis. Circ Res 2018;122:97-105.

139. Selwaness M, Bouwhuijsen QJAvd, Verwoert GC, et al. Blood Pressure Parameters and Carotid Intraplaque Hemorrhage as Measured by Magnetic Resonance Imaging. Hypertension 2013;61:76-81.

140. Mujaj B, Bos D, Kavousi M, et al. Serum insulin levels are 
associated with vulnerable plaque components in the carotid artery: the Rotterdam Study. Eur J Endocrinol 2020.

141.Pletsch-Borba L, Selwaness M, van der Lugt A, et al. Change in Carotid Plaque Components: A 4-Year FollowUp Study With Serial MR Imaging. JACC Cardiovasc Imaging 2018;11:184-92.

142. Underhill HR, Yuan C, Yarnykh VL, et al. Arterial remodeling in [corrected] subclinical carotid artery disease. JACC Cardiovasc Imaging 2009;2:1381-9.

143.Mujaj B, Bos D, Selwaness M, et al. Statin use is associated with carotid plaque composition: The Rotterdam Study. Int J Cardiol 2018;260:213-8.

144. Takaya N, Yuan C, Chu B, et al. Presence of intraplaque hemorrhage stimulates progression of carotid atherosclerotic plaques: a high-resolution magnetic resonance imaging study. Circulation 2005;111:2768-75.

145. Kwee RM, Truijman MTB, van Oostenbrugge RJ, et al. Longitudinal MRI study on the natural history of carotid artery plaques in symptomatic patients. PLoS One 2012;7:e42472.

146. Underhill HR, Yuan C, Zhao XQ, et al. Effect of rosuvastatin therapy on carotid plaque morphology and composition in moderately hypercholesterolemic patients: a high-resolution magnetic resonance imaging trial. Am Heart J 2008;155:584.e1-8.

147. Alkhalil M, Biasiolli L, Akbar N, et al. T2 mapping MRI technique quantifies carotid plaque lipid, and its depletion after statin initiation, following acute myocardial infarction. Atherosclerosis 2018;279:100-6.

148. Saam T, Yuan C, Chu B, et al. Predictors of carotid atherosclerotic plaque progression as measured by noninvasive magnetic resonance imaging. Atherosclerosis

Cite this article as: Kassem M, Florea A, Mottaghy FM, van Oostenbrugge R, Kooi ME. Magnetic resonance imaging of carotid plaques: current status and clinical perspectives. Ann Transl Med 2020;8(19):1266. doi: 10.21037/atm-2020-cass-16 2007;194:e34-42.

149. Yuan C, Oikawa M, Miller Z, et al. MRI of carotid atherosclerosis. J Nucl Cardiol 2008;15:266-75.

150.Kerwin WS, Hatsukami T, Yuan C, et al. MRI of carotid atherosclerosis. AJR Am J Roentgenol 2013;200:W304-13.

151.Meschia JF, Bushnell C, Boden-Albala B, et al. Guidelines for the primary prevention of stroke: a statement for healthcare professionals from the American Heart Association/American Stroke Association. Stroke 2014;45:3754-832.

152.Endarterectomy for asymptomatic carotid artery stenosis. Executive Committee for the Asymptomatic Carotid Atherosclerosis Study. JAMA 1995;273:1421-8.

153. Halliday A, Mansfield A, Marro J, et al. Prevention of disabling and fatal strokes by successful carotid endarterectomy in patients without recent neurological symptoms: randomised controlled trial. Lancet 2004;363:1491-502.

154. Chambers BR, Donnan G. Carotid endarterectomy for asymptomatic carotid stenosis. Cochrane Database Syst Rev 2005;(4):CD001923.

155. Naylor AR, Ricco JB, de Borst GJ, et al. Editor's Choice \&\#x2013; Management of Atherosclerotic Carotid and Vertebral Artery Disease: 2017 Clinical Practice Guidelines of the European Society for Vascular Surgery (ESVS). Eur J Vasc Endovasc Surg 2018;55:3-81.

156. Yuan C, Parker DL. Three-Dimensional Carotid Plaque MR Imaging. Neuroimaging Clin N Am 2016;26:1-12.

157. Balu N, Zhou Z, Yuan C. Vessel Wall Imaging in the Era of Artificial Intelligence. In: Yuan C, Hatsukami TS, Mossa-Basha M. Editors. Vessel Based Imaging Techniques. Springer, 2020:283-94. 